\title{
Expression Profiling of Osteosarcoma Cells Transfected with MDR1 and NEO Genes: Regulation of Cell Adhesion, Apoptosis, and Tumor Suppression-Related Genes
}

\author{
Marta Sanchez-Carbayo, Thomas J. Belbin, Katia Scotlandi, Michael Prystowsky, \\ Nicola Baldini, Geoffrey Childs, and Carlos Cordon-Cardo \\ Memorial Sloan-Kettering Cancer Center (MS-C, CC-C), Albert Einstein College of Medicine (TJB, MP, GC), New \\ York, New York; and Instituti Ortopedici Rizzoli (KS, NB), Bologna, Italy
}

\begin{abstract}
SUMMARY: The expression patterns of the osteosarcoma cell line U-2 OS, and three derived subclones containing stably transfected MDR1, NEO and MDR1/NEO genes were compared using cDNA microarrays comprising 8976 known genes and expressed sequenced tags. Data provided new insights into three critical issues. First, MDR1 overexpression was associated with altered expression of genes related to several cellular pathways, including (a) drug influx/efflux (eg, dynamin 3), (b) metabolic enzymes (eg, monoamine oxidase A), (c) cell adhesion (eg, EPCAM), (d) apoptotic signaling (eg, I-TRAF), (e) senescence (eg, telomerase RNA binding protein staufen), (f) tumor suppression-related genes (eg, KISS-1 and ephrin B3), and (g) immune system receptors (eg, LENG2). MDR1, EPCAM, and ephrin B3 expression was confirmed by immunohistochemistry. Second, MDR1 transfected cells selected with either doxorubicin or neomycin showed distinct expression profiles that could be related to differential selection. Moreover, hierarchical clustering indicated that cells transfected with MDR1 alone, or cotransfected with $N E O$, displayed more closely related expression profiles than cells transfected only with NEO. Third, transfection with NEO and selection with neomycin produced a considerable number of expression changes within the cell. This study further elucidates the genetic events associated with MDR1 expression and identifies novel targets associated with multidrug resistance. (Lab Invest 2003, 83:507-517).
\end{abstract}

\begin{abstract}
P-glycoprotein (Pgp) is a $170 \mathrm{kd}$ ATP-dependent 1 transmembrane protein encoded by the MDR1 gene involved in cellular transport mechanisms and reduced intracellular drug accumulation (Gottesman and Pastan, 1993). Gene transfer experiments have demonstrated that Pgp expression is sufficient for the establishment of the multidrug resistance (MDR) phenotype that certain tumor cells display to chemotherapeutic drugs (Gottesman and Pastan, 1993; Ueda et al, 1987). However, neither the precise physiologic function of Pgp nor the mechanism by which it induces the MDR phenotype is completely known. Several molecular mechanisms have been associated with the clinical phenomenon of MDR, including alterations of genes involved in intracellular transport, DNA repair, metabolism, cell adhesion, and apoptotic signaling (Damiano et al, 1999; Desoize et al, 1998; Johnstone et al, 1999; St. Croix and Kerbel, 1997). More recently, induction of MDR has been associated with simultaneous alterations in expression of many of these critical genes (Kudoh et al, 2000). However, the
\end{abstract}

\section{DOI: 10.1097/01.LAB.0000064702.63200.94}

Received September 26, 2002.

Address reprint requests to: Dr. Marta Sanchez-Carbayo, Division of Molecular Pathology, Memorial Sloan-Kettering Cancer Center, 1275 York Avenue, New York, New York 10021. E-mail: cordon-c@mskcc.org potential involvement of the MDR1 gene in their simultaneous expression has not been reported to date.

To further elucidate the molecular mechanisms linked to Pgp mediated MDR, cDNA microarrays were used to study the expression patterns of closely related osteosarcoma cell clones engineered to stably express Pgp, the neomycin resistance gene (NEO), or Pgp plus NEO (Scotlandi et al, 1999). Osteosarcoma cells represent a dynamic model because resistant clones have been shown to display a less aggressive behavior both in vitro and in vivo with respect to parental cells (Chan et al, 1997; Scotlandi et al, 1996; Wunder et al, 2000). Moreover, because some of these isogenic cells have not been exposed to any previous treatment, they can be considered as pure transfectants, and any genotypic/gene expression alteration could be attributed directly or indirectly to MDR1/ NEO genes or selection with doxorubicin/neomycin.

\section{Results}

Differences in expression profiles among derived clones and the isogenic parental cell line were studied in five independent experiments, as shown in the experimental design (Fig. 1). Out of 8976 genes spotted onto the array, 3987 provided data simultaneously in the three derived MDR1 or NEO transfected clones. Hierarchical clustering analysis (Eisen et al, 1998) 


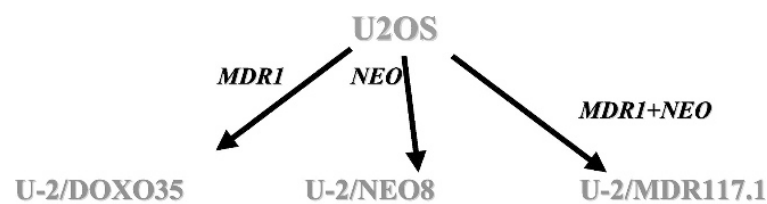

U-2/DOX035

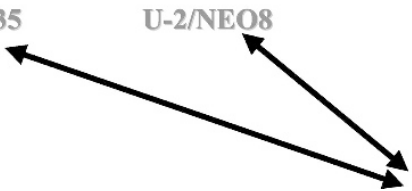

$-2 / M D R 117.1$

Figure 1.

Scheme of the experimental design for the transfection and cotransfection of cell lines with NEO or MDR1 genes. Five analyses were performed. First, U-2 OS parental cell line was labeled with Cy3 and compared with each of its derived clones labeled with Cy5: U-2/DOX035 (obtained by transfection of MDR1 and selection with doxorubicin), U-2/NE08 (transfected with NEO and selected with G418), and U-2/MDR117.1 (cotransfected with MDR1/NEO and selected with G418). Second, U-2/MDR117.1 RNA was labeled with Cy5 and compared with either U-2/NE08 or U-2/DOX035 clones; these latter were dyed with Cy3.

indicated that MDR1-transfected clones displayed more similarities to each other than the NEOengineered clones (Fig. 2A).

\section{Differentially Expressed Genes in MDR1 Transfected Clones}

Genes Associated with MDR1 Overexpression Alone

Genes Differentially Expressed in U-2/DOXO $35 \mathrm{Ver}$ sus U-2 OS and U-2/MDR117.1 Versus U-2/NEO8 Cell Clones. The only overexpressed gene in these experiments was MDR1. Expression of MDR1 was confirmed at the RNA level by Northern blot (Fig. 2B; Table 1) and at the protein level by immunohistochemistry (Fig. 2C). We observed an increased MDR1 expression in U-2/DOXO35, the cells selected with doxorubicin as compared with U-2/MDR117.1 and the cells selected with neomycin, by cDNA microarrays, Northern blot, and immunohistochemistry. This suggests that in addition to the direct effect of the transfection, the selection agent doxorubicin can induce MDR1 expression. Underexpressed genes included staufen and EPCAM. We confirmed the loss of expression of EPCAM in MDR1 transfected clones by immunohistochemistry (Fig. 3A).

Genes Expressed in U-2/MDR117.1 Relative to U-2/ NEO8 and in U-2/MDR117.1 Relative to U-2 OS Cell Clones. Overexpressed genes common to both experiments included MDR1 and histone 4. Underexpressed genes included other adhesion-related targets (eg, protocadherin 13), the immune receptor cluster LENG2, and the apoptosis-related I-TRAF gene.

Genes Differentially Expressed Only in U-2/MDR117.1 Versus U-2/NEO8 Cell Clones. Enzymes such as sialyltransferase and mannose phosphate isomerase were overexpressed in U-2/MDR117.1 cells when compared with cells transfected with NEO alone. Genes underexpressed included metabolic enzymes, signal transduction related genes, other adhesion-related molecules such as protocadherin- $\alpha$ and zyxin, several membrane transport proteins, and the endocytotic protein dynamin 3.

Genes Associated with Synergy between MDR1 and Doxorubicin. The genes differentially expressed only in the experiment evaluating U-2/DOXO35 versus $U-2$ OS cell clones could be considered either related to MDR1 or doxorubicin treatment (Table 1). Overexpressed genes included certain tumor-progression related genes such as the human malignant melanoma metastasis-suppressor KISS-1, other metabolic enzymes, and a gene involved in cell-surface transport; HIV-1 induced protein $\mathrm{HIN}-1$. We have confirmed the expression of KISS-1 by semiquantitative PCR in these clones (Fig. 3B). Underexpressed genes included the intracellular $\mathrm{pH}$ regulator ATP synthase $\mathrm{H}+$ transporting mitochondrial complex, other tumor progression related genes such as WDNM-1, other transport endocytosis-related genes such as the mosaic protein LR11, and cell-growth FGF7 genes.

Genes Associated with Additive Interactions between MDR1 and Neomycin. The genes differentially expressed only in the experiment evaluating $\mathrm{U}-2$ / MDR117.1 versus U-2 OS cell clones could be considered related to both MDR1 and neomycin treatment (Table 1). MDR1/NEO cotransfection resulted in overexpression of a group of genes not affected in U-2/ NEO8 or U-2/DOXO35 cells when compared with the parental cell line. Examples of such changes include a signal transduction gene, specifically a Rac1 associated protein (Sra-1). Among the underexpressed genes were other apoptotic pathway genes such as advillin, and tumor progression associated genes such as the proto-oncogene c-mer.

Genes Associated with Synergy between MDR1 and Doxorubicin and Neomycin. Two genes that were commonly and differentially expressed only in the experiments comparing $\mathrm{U}-2 / \mathrm{DOXO} 35$ versus $\mathrm{U}-2$ OS and $U-2 / N E O 8$ versus $U-2$ OS cells can be considered to be associated with MDR1, doxorubicin, or neomycin (Table 1). Only the TAR RNA (HIV) binding protein (TRBP2) was overexpressed and the heat-shock protein HSP 90 gene underexpressed in this category.

\section{Differentially Expressed Genes in NEO Transfected Clones}

Genes Activated with Neomycin Selection Alone. The genes differentially expressed in U-2/NEO8 relative to $\mathrm{U} 2-\mathrm{OS}$ and in U-2/MDR117.1 relative to U2-OS and unchanged in U-2/DOXO35 cells could be considered associated with neomycin selection. These include overexpression of keratin 5 and underexpression of protoporphyrinogen oxidase. Second, those genes differentially expressed only in U-2/NEO8 versus U-2 OS cells should also be considered. Transfection of cells with NEO resulted in expression changes of genes related to transcription, polyamines, calcium and lipid metabolism, signal transduction, and differentiation. The microarray comparing the NEO transfected clone versus the parental cells was considered a critical control in the 

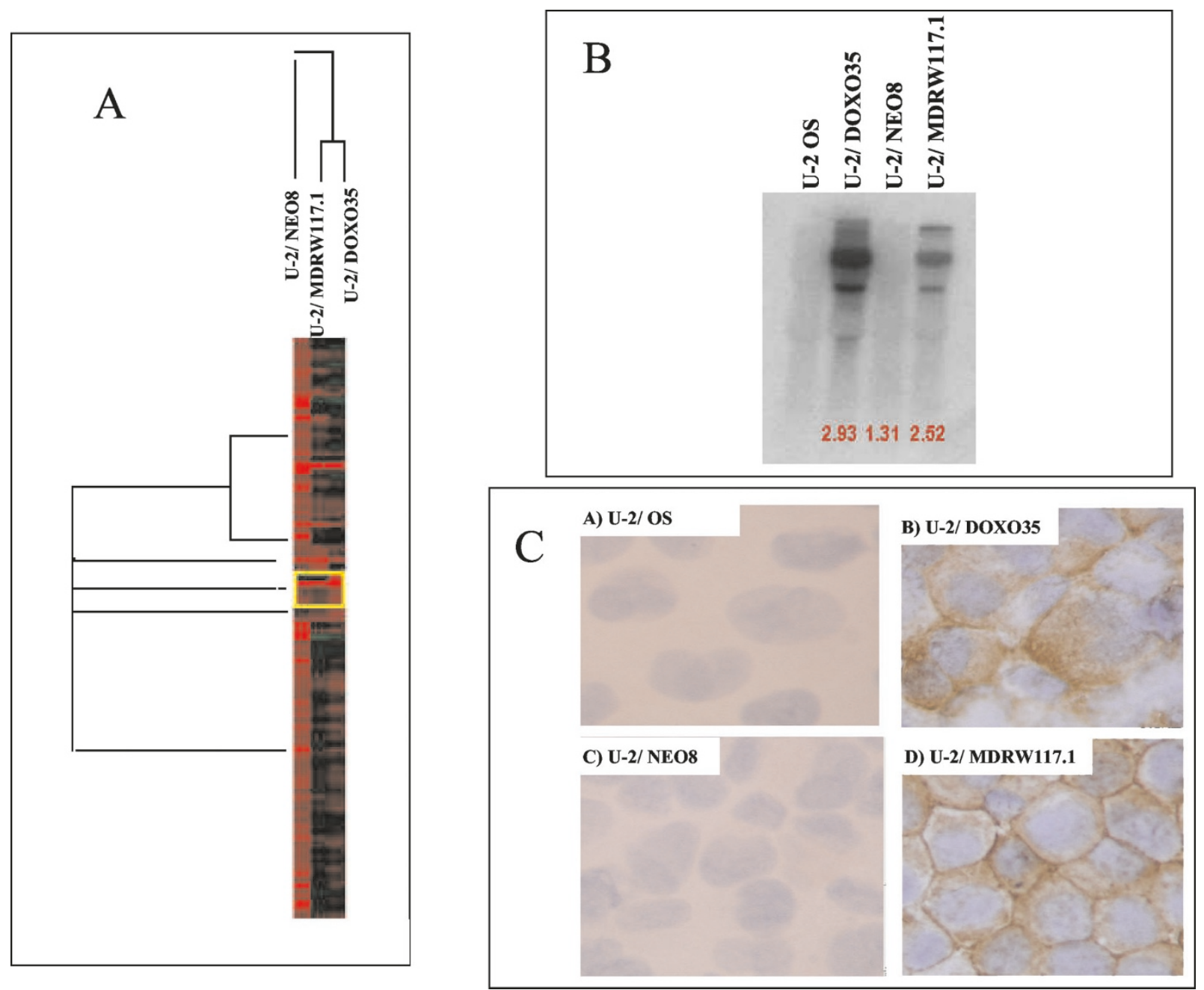

\section{Figure 2.}

A, Clustering tree-view analysis of the transfected cell lines versus the parental U-2 OS considering those 226 genes showing red/green (R/G) ratios of 2-fold or higher in at least one experiment. MDR1 transfected and cotransfected clones U-2/DOX035 and U-2/MDR117.1, respectively, were more closely related than U-2/NE08 transfected clones U-2/NE08 and U-2/MDR117.1. B, Northern blot of MDR1 gene. C, Immunohistochemical staining of Pgp using Hyb241 antibody in the four cell lines. No band for MDR1 in northern blots and no positive staining for Pgp in immunohistochemistry were observed in U-2 OS and U-2/NE08 cells as compared with $\mathrm{U}-2 / D 0 X 035$ and U-2/MDR117.1. These observations confirmed an effective transfection/cotransfection of the MDR1 gene. Note how the cDNA microarray R/G ratio was smaller than in the Northern blot as a result of the dilution of the RNA during hybridization. Although total RNA is hybridized against one specific probe in the Northern blot, thousands of cDNAs are hybridized to respective retrotranscribed total RNA (Taniguchi et al, 2001; Yue et al, 2001).

experimental design. An optimal hybridization was confirmed throughout the dynamic range (Fig. 4).

Genes Associated with Synergy Between Neomycin and MDR1. The genes expressed in both U-2/NEO8 versus $U-2$ OS and U-2/MDR117.1 versus U-2/NEO8 cell clones could be considered associated with either MDR1 or neomycin treatment (Table 1). Overexpressed genes included the BCL-2 athanogene (BAG4) and the NKG-2 type II integral membrane protein gene. Underexpressed genes included Not56like tumor-related gene.

Second, the genes expressed in U-2/NEO8 versus $\mathrm{U}-2$ OS in U-2/MDR117.1 versus $\mathrm{U}-2$ /NEO8 and in U-2/MDR117.1 versus U-2 OS cells could also be considered associated with either MDR1 or neomycin treatment. Overexpression was found for polymerase (RNA) II (DNA directed) polypeptide A. Underexpressed genes included metabolic-, differentiation-, adhesion-, and signaling-related genes. Because these genes were not present in U-2/DOXO35 and $\mathrm{U}-2 / \mathrm{NEO} 8$, and U-2/MDR117.1 clones were selected with neomycin, these genes could be associated with neomycin selection alone. However, because they were present in U-2/MDR117.1 versus U-2/NEO8, it could not be ruled out that the expression of these genes is MDR1 related.

\section{Comparison of Expression Profiles of MDR Cell Clones}

We independently analyzed the differentially expressed genes between MDR1 transfected (U-2/ DOXO35) versus MDR1/NEO cotransfected (U-2/ MDR117.1) cells (Table 2). Genes highly expressed in U-2/MDR117.1 include enzymes involved in drug metabolism, immune response targets, and tumor related genes such as ephrin B3. We have confirmed the differential expression of ephrin B3 in U-2/DOXO35 and U-2/MDR117.1 by immunohistochemistry (Fig. 5). 
Table 1. Differentially Expressed Genes Among MDRI Transfected, NEO Transfected, and MDRI/NEO Co-Transfected U-2 Cells

\begin{tabular}{|c|c|c|c|c|c|}
\hline Gene Name & $\begin{array}{l}\text { Accession } \\
\text { Number }\end{array}$ & $\begin{array}{l}\text { U-2/D0X035 } \\
\text { VS U-2 OS }\end{array}$ & $\begin{array}{l}\text { U-2/NE08 } \\
\text { VS U-2 OS }\end{array}$ & $\begin{array}{l}\text { U-2/MDR117.1 } \\
\text { VS U-2/OS }\end{array}$ & $\begin{array}{l}\text { U-2/MDR117.1 } \\
\text { VS U-2 NE08 }\end{array}$ \\
\hline Multidrug resistance protein 1 & AA455911 & 2.93 & 1.31 & 2.52 & 4.1 \\
\hline Connector enhancer suppressor of ras (CNK1) & AA459278 & 2.06 & 1.784 & 1.46 & 1.77 \\
\hline Galactoside alpha-2,3-sialyltransferase & H19227 & 1.97 & 0.90 & & 4.19 \\
\hline Mannose phosphate isomerase & AA482198 & 1.31 & 1.77 & 1.53 & 2.33 \\
\hline CD8 antigen, alpha polypeptide (p32) & AA443649 & 1.36 & 1.519 & 1.89 & 2.25 \\
\hline Cystatin M & W72895 & 1.25 & 1.396 & 0.95 & 0.4 \\
\hline Dynamin 3 & T67523 & 1.21 & 1.21 & 0.95 & 0.5 \\
\hline $\begin{array}{l}5 \text { methyltetrahydrofolate-homocysteine } \\
\text { methyltransferase (MTR) }\end{array}$ & AA233650 & 1.15 & 1.18 & 1.06 & 0.31 \\
\hline Protocadherin alpha & H16743 & 1.07 & 1.19 & 0.63 & 0.48 \\
\hline $\mathrm{H} 4$ histone & AA868008 & 0.99 & 1.54 & 2.29 & 2.37 \\
\hline LENG2 & N46968 & 0.95 & 0.96 & 0.47 & 0.46 \\
\hline Protocadherin 13 & AA040043 & 0.83 & 0.74 & 0.45 & 0.39 \\
\hline Integrin, beta 3 & AA037229 & 0.77 & 0.82 & 0.58 & 0.47 \\
\hline G-protein coupled receptor for UDP-Glucose & AA027011 & 0.75 & 0.88 & 0.55 & 0.44 \\
\hline Phosphofructokinase, muscle & AA099169 & 0.70 & 0.96 & 0.59 & 0.5 \\
\hline Zyxin & AA047443 & 0.67 & 0.76 & 0.51 & 0.39 \\
\hline Zinc/cadmium resistance protein & AA134753 & 0.65 & 1.04 & 0.51 & 0.44 \\
\hline TRAF-interacting protein I-TRAF & AA134814 & 0.65 & 0.94 & 0.37 & 0.4 \\
\hline Matrix metalloproteinase 7 (PUMP-1) & AA031513 & 0.64 & 0.72 & 0.42 & 0.37 \\
\hline Sodium phosphate transporter (NPT3) & H60423 & 0.60 & 1.24 & 0.7 & 0.46 \\
\hline Brain-derived neurotrophic factor & AA262988 & 0.59 & 0.94 & 0.52 & 0.39 \\
\hline Kinesin heavy chain & AA046690 & 0.52 & 0.59 & 0.52 & 0.39 \\
\hline EPCAM & AA454810 & 0.49 & 0.63 & 0.46 & 0.41 \\
\hline Staufen (STAU2) & AA045462 & 0.49 & 0.80 & 0.42 & 0.35 \\
\hline Malignant melanoma metastasis-suppressor (KiSS-1) & AA464595 & 2.80 & 1.22 & 0.99 & 1.01 \\
\hline Tax interaction protein 43 & R10726 & 2.34 & 0.89 & 0.53 & 0.86 \\
\hline Monoamine oxidase $\mathrm{A}$ & AA011096 & 2.14 & 1.38 & 1.12 & 0.96 \\
\hline Putative HIV-1 induced protein HIN-1 & AA902831 & 2.12 & 1.08 & 1.09 & 1.02 \\
\hline APOL gene for apolipoprotein L & AA457728 & 2.03 & 1.119 & 0.7 & \\
\hline Mosaic protein LR11 & AA487543 & 0.43 & 1.17 & 0.99 & 1.06 \\
\hline Fibroblast growth factor 7 & AA009609 & 0.43 & 0.76 & 1.34 & 1.09 \\
\hline $\begin{array}{l}\text { ATP synthase, } \mathrm{H}+\text { transport, mitochondrial FO } \\
\text { complex, subunit b }\end{array}$ & AA453849 & 0.40 & 1.30 & 0.86 & 0.82 \\
\hline $\begin{array}{l}\text { Methyl-CpG-binding protein (MeCP-2) (Rett } \\
\text { syndrome) }\end{array}$ & AA977094 & 0.37 & 1.05 & 0.82 & 0.9 \\
\hline WDNM1 & AA149250 & 0.39 & 1.482 & 0.64 & 0.83 \\
\hline Specifically Rac1-associated protein (Sra-1) & AA598583 & 1.24 & 1.21 & 2.39 & 1.05 \\
\hline Polymerase (RNA) II (DNA directed) polypeptide A & AA479052 & 1.86 & 2.756 & 2.39 & 3.01 \\
\hline BCL2 athanogene 4 (BAG4) & N25897 & 1.68 & 2.96 & 1.53 & 2.17 \\
\hline NKG2-D type II integral membrane protein & ААЗ97819 & 1.69 & 2.396 & 1.53 & 2.06 \\
\hline $\begin{array}{l}\text { Prolyl 4-hydroxylase alpha (II) subunit, } \\
\text { procollagen-proline }\end{array}$ & W49522 & 0.74 & 0.359 & 0.61 & 0.5 \\
\hline Not56-like protein & R23251 & 1.07 & 0.195 & 0.55 & 0.49 \\
\hline Neurotransmitter transporter (NTT5) & AA005387 & 1.09 & 1.26 & 0.5 & 0.87 \\
\hline Human cellular proto-oncogene (c-mer) & AA436591 & 0.76 & 0.69 & 0.5 & 0.51 \\
\hline Human retinoic acid-responsive protein (NN8-4AG) & AA934704 & 0.73 & 0.97 & 0.49 & 0.68 \\
\hline Glutamate receptor, metabotropic 3 & AA670430 & 1.03 & 1.37 & 0.48 & 0.94 \\
\hline Human tumor susceptibility protein (TSG101) & AA670215 & 0.77 & 0.288 & 0.48 & 0.48 \\
\hline Brain-cadherin & AA418564 & 0.54 & 0.486 & 0.44 & 0.41 \\
\hline $\begin{array}{l}\text { Dual specificity phosphatase } 11 \text { (RNA/RNP) complex } \\
1 \text { interacting }\end{array}$ & AA463480 & 0.55 & 0.388 & 0.43 & 0.42 \\
\hline UDP-glucuronosyltransferase 2B4 precursor & N53031 & 0.75 & 0.152 & 0.4 & 0.38 \\
\hline TRAM-like protein & T74606 & 0.99 & 1.135 & 0.38 & 0.93 \\
\hline OB-cadherin-2 & AA136983 & 0.52 & 0.314 & 0.35 & 0.26 \\
\hline Advillin & AA427733 & 1.03 & 1.07 & 0.27 & \\
\hline Human TAR (HIV) RNA-binding protein 2 (TARBP2) & AA436409 & 2.01 & 2.79 & 1.91 & 1.65 \\
\hline Heat shock protein HSP 90 ALPHA & H88540 & 0.45 & 0.27 & 0.76 & 1.12 \\
\hline
\end{tabular}




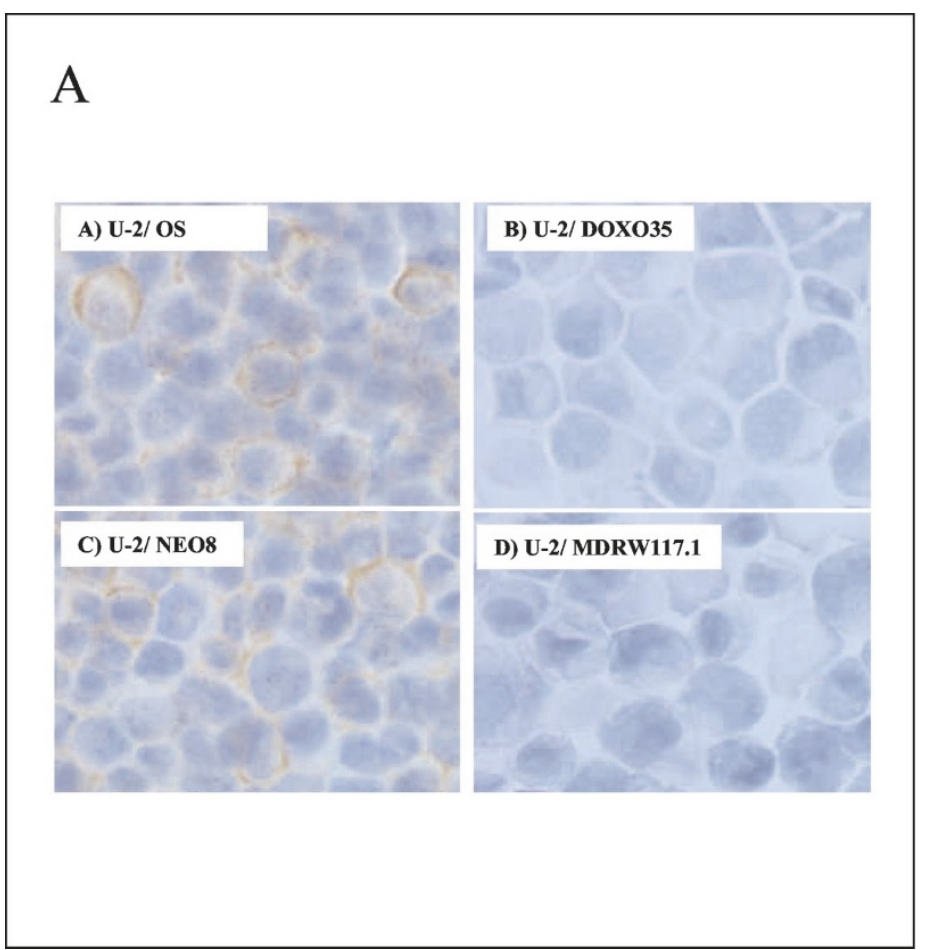

B

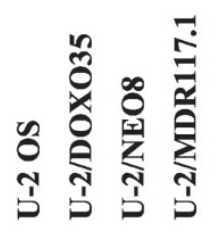

Kiss-1

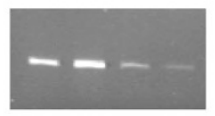

202-bp

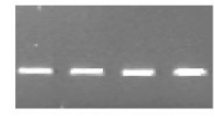

650-bp

$\beta$-actin

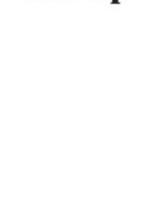

\section{Figure 3.}

A, Immunohistochemical staining of EPCAM in four cell clones highlighting the loss of immunoreactivities observed in the U-2/D0X035 cells and U-2/MDRW117.1 cells as compared with positive immunostaining observed in the U-2 OS and U-2/NE08 cells, confirming the expression profiling results of the microarray. B, Semiquantitative RT-PCR analysis of KISS-1 expression in U-2 OS parental cells and MDR transfectants highlighting the difference in expression levels between U-20S and U-2/DOXO 35 cells. Total RNA was reverse transcribed, and amplified products were analyzed after 27 cycles by electrophoresis in $2 \%$ agarose gel.
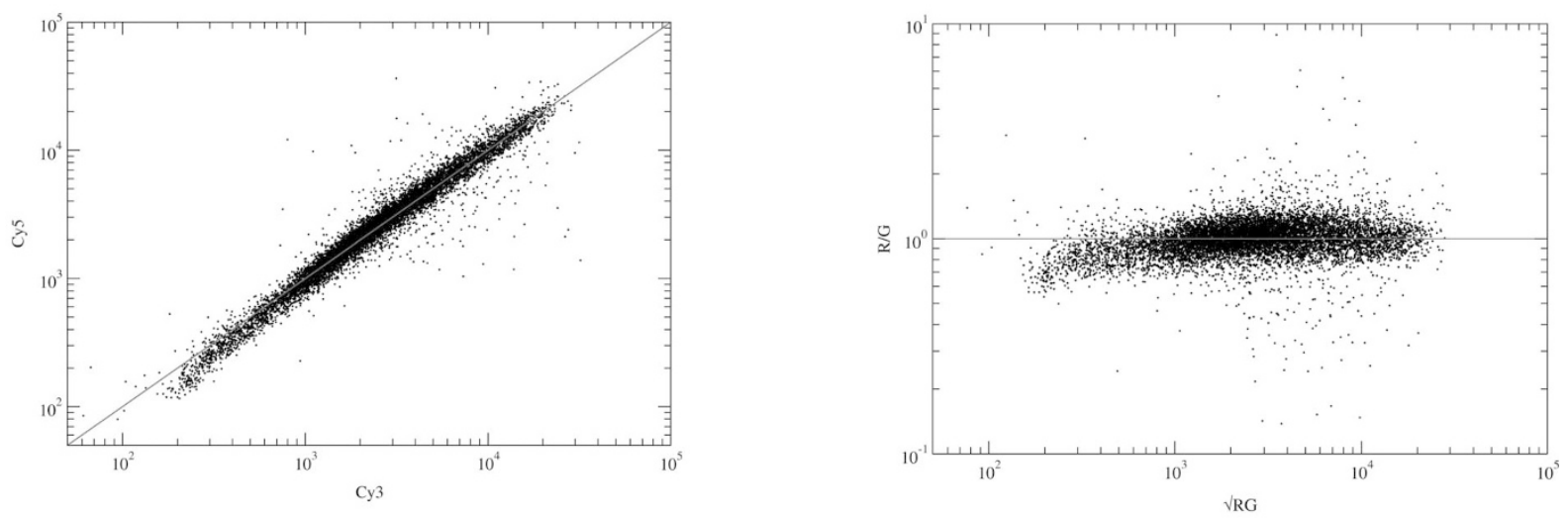

Figure 4.

Scatter plots of U-2/NE08 (Cy5) versus U2-OS (Cy3) experiment. A, Cy5 and Cy3 dyes were balanced taking a normalization factor of 1.13. B, Dispersion of ratios of the dyes along the dynamic range of the square root of the product of the intensities of both dyes. It is common to plot a red versus green channel scatter plot to examine distribution of intensities; however, we found that transforming to fold change versus average intensity displayed the data in a more easily viewed form. The curvature in the scatter plot indicated a dependence on the ratio $\mathrm{R}$ on the overall intensity. Optimal normalized data should be horizontal and centered at 0 . Samples were normalized using this intensity-dependent normalization using the Splus function lowess (Callow et al, 2000), which is more accurate than normalization with a fixed constant (Eisen et al, 1998).

Highly expressed genes in U-2/DOXO35 include transmembrane proteins and immune system involved receptors such as interferon gamma receptor 2 .

\section{Comparison of Expression Profiles of Different Subclones}

To rule out the possibility of clonal variation either produced by cultured cells undergoing somatic mutations during prolonged in vitro periods or epigenetic mechanisms, we verified the expression changes of MDR1 in parallel clones with different MDR1 expres- sion levels (Scotlandi et al, 1999). We have compared the expression profiling of two subclones of the MDR1 transfection (U-2/DOXO23 and U-2/DOXO35) and two subclones of the MDR1/NEO cotransfection (U-2/ MDR117.1 and U-2/MDR117.2). We searched for genes differentially expressed between each pairs of MDR1 transfected and MDR1/NEO cotransfected clones. We did not observe differences higher than the 2-fold cutoff between these clones regarding the MDR1 related genes identified and outlined above 
Table 2. Differentially Expressed Genes Between MDRI/NEO Co-Transfected and MDRI Transfected U-2 Cells

\begin{tabular}{|c|c|c|}
\hline Gene Name & $\begin{array}{l}\text { Accession } \\
\text { Number }\end{array}$ & $\begin{array}{c}\text { U-2/MDR117.1 } \\
\text { VS U-2/D0X035 }\end{array}$ \\
\hline Glutathione S-transferase subunit 4 & AA290737 & 3 \\
\hline H4 histone & AA868008 & 2.74 \\
\hline MHC class II DQ-beta associated with DR2, DQw1 protein & AA442984 & 2.51 \\
\hline Connector enhancer suppressor of ras (CNK1) & AA459278 & 2.48 \\
\hline Oncostatin-M specific receptor beta subunit (OSMRB) & AA909184 & 2.47 \\
\hline CD8 antigen, alpha polypeptide (p32) & AA443649 & 2.46 \\
\hline Ephrin-B3 & AA485795 & 2.42 \\
\hline Proteasome zeta chain & AA598815 & 2.37 \\
\hline NKG2-D type II integral membrane protein & AA397819 & 2.13 \\
\hline BCL2 athanogene 4 (BAG4) & N25897 & 2.08 \\
\hline MHC class II HLA-DR2-Dw12 mRNA DQw1-beta & AA458472 & 2.07 \\
\hline Interferon gamma receptor 2 (interferon gamma transducer 1) (IFNGR2) & AA448929 & 0.5 \\
\hline SRB7 (suppressor of RNA polymerase B7) & AA130736 & 0.49 \\
\hline Brain-derived neurotrophic factor & AA262988 & 0.49 \\
\hline Transmembrane protein with EGF-like and 2 follistatin-like domains 1 (TMEFF1) & AA431678 & 0.48 \\
\hline Matrix metalloproteinase 7 (PUMP-1) & AA031513 & 0.47 \\
\hline Protocadherin 13 & AA040043 & 0.45 \\
\hline Malignant melanoma metastasis-suppressor (KiSS-1) & AA464595 & 0.42 \\
\hline Prolyl 4-hydroxylase alpha (II) subunit, procollagen-proline & W49522 & 0.42 \\
\hline Actin filament associated protein (AFAP) & R69355 & 0.41 \\
\hline LENG2 & N46968 & 0.40 \\
\hline Kinesin heavy chain & AA046690 & 0.40 \\
\hline UDP-glucuronosyltransferase 2B4 precursor & N53031 & 0.40 \\
\hline Not56-like protein & R23251 & 0.39 \\
\hline Brain-cadherin & AA418564 & 0.35 \\
\hline OB-cadherin-2 & AA136983 & 0.31 \\
\hline EPCAM & AA454810 & 0.29 \\
\hline
\end{tabular}

\section{$\mathrm{U}-2 / \mathrm{DOX} 035$}

\section{U-2/MDRW117.1}
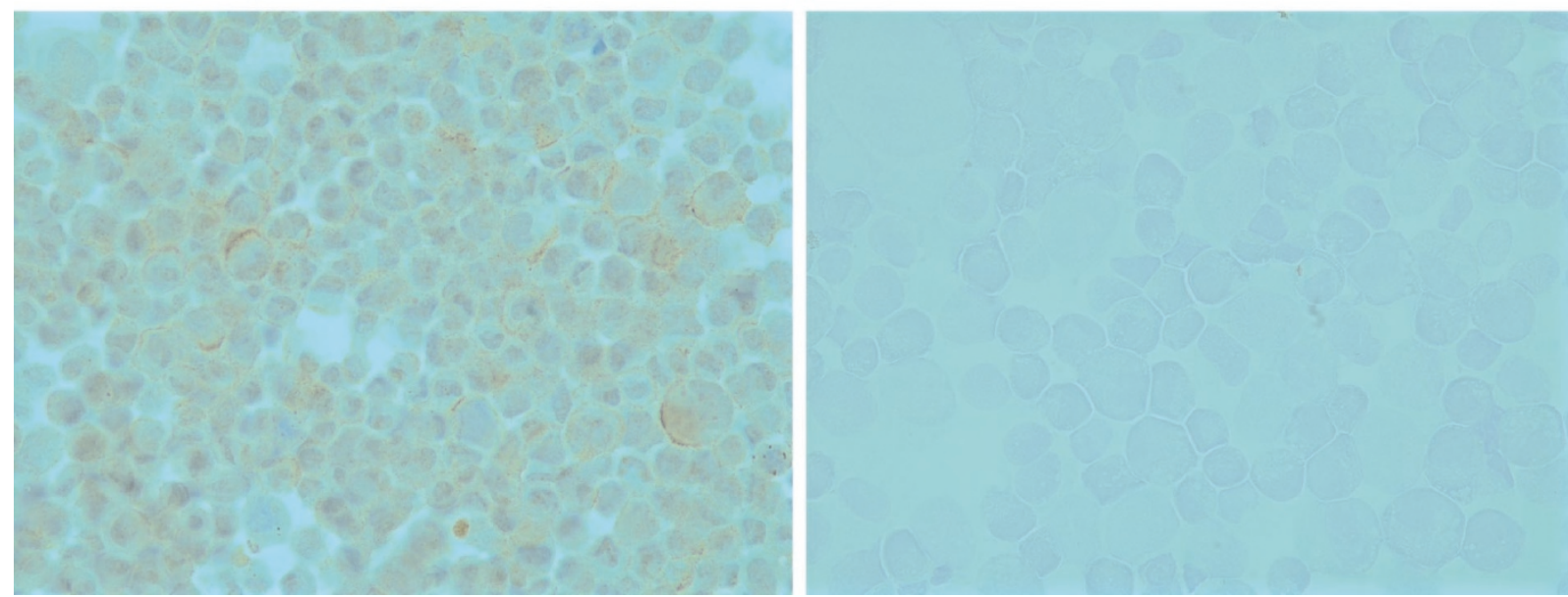

Figure 5.

Immunohistochemical staining of ephrin B3 in U-2/DOX035 and U-2/MDR117.1 resistant cell lines highlighting the diffuse immunoreactivities observed in the U-2/D0X035 cells and the undetectable levels observed for U-2/MDR117.1 cells, confirming the expression profiling results of the microarrays.

(www.mskcc.org/GCL/SarcomaGenomics). The expression of MDR1 was confirmed to have a lower expression in U-2/DOXO23 as compared with $\mathrm{U}-2 /$ DOXO35 as well as in U-2/MDR117.2 as compared with U-2/MDR117.1 in these experiments. These observations were confirmed by the analysis of two additional microarray experiments comparing U-2/ DOXO23 versus U-2 OS and U-2/MDR117.2 versus 
$\mathrm{U}-2 / \mathrm{NEO}$. The complete list of differential genes observed in these validation experiments will be available as supplementary data on our web site.

\section{Discussion}

The discovery of the drug-resistance phenotype as an exclusive feature of tumor cells led to the consideration of MDR as a pleiotropic oncogenic phenomenon. With genetic and biochemical evidence that the MDR1 gene encodes Pgp, a transmembrane transporter (Gottesman and Pastan, 1993; Ueda et al, 1987), the MDR phenotype was initially modeled as a drug-pump mechanism (Dano et al, 1973). The observation that Pgp was expressed and functional in certain normal cells, mainly those associated with secretory and blood-tissue barrier functions, revealed a physiologic role for Pgp. Although the precise function of Pgp remains undetermined, its major role appears to be related to the protection of the cells against toxic xenobiotics to which they may be exposed. Mdr1-null mice were viable, fertile, and did not display obvious phenotypic abnormalities other than hypersensitivity to drugs and neurotoxicity (Schinkel et al, 1997). The analysis of these knockout mice produced genetic proof that lack of MDR1 function was not a lethal but a pleiotropic event (Desoize et al, 1998). Given the inability of the drug-pump model to explain several important MDR phenomena, alternatives such as the altered-partitioning model have been proposed. Its basic principle is that MDR proteins might not directly transport drugs, but that their expression leads to altered regulation of ion transport or signal transduction (Roepe, 2000).

Data from our analyses provide new insights into three critical issues. First, MDR1 expression is associated with changes in several important cellular pathways, potentially involved in the MDR phenotype, which have not been previously reported as a consequence of MDR1 transfection. Second, MDR1 transfected cells selected with either doxorubicin or neomycin showed distinct expression profiles that could be related to differential induction or treatment selection. Interestingly, the differences between these clones might also account for their distinct degrees of resistance (Scotlandi et al, 1999). Finally, transfection with the NEO gene and selection with neomycin produced a considerable number of expression changes within the cell. This is a significant finding because $N E O$ is a standard control in transfection experiments.

MDR1 transfection affected the expression of genes reported to be associated with the lower concentration of anticancer drugs in MDR cells (Dano, 1973; Roepe, 2000). MDR1 transfection resulted in underexpression of membrane proteins involved in active transport, such as the UDP-glucose transporter (Chambers et al, 2000) and the sodium phosphate transporter (Tenenhouse, 1999) as well as endocytic transport genes such as dynamin (Modregger et al, 2000) and mosaic protein LR11 (Hirayama et al, 2000). These findings support an involvement of MDR1 in drug influx by mechanisms indirectly mediated by Pgp. Enzymatic metabolism was modified as well. This, in turn, may alter intracellular toxicity to certain anticancer drugs or xenobiotics. The association of MDR1 and proteasome enzymes has not been documented previously, which supports the new therapeutic efforts targeting this complex by proteasome inhibitors (Adams et al, 2000).

This study also expands the already described alterations of certain adhesion proteins, such as integrins, in U-2/DOXO35 cells (Scotlandi et al, 1999). Genes such as osteoblast cadherins (Okazaki et al, 1994), EPCAM (Trebak et al, 2001), zyxin (Hirota et al, 2000), and specific metalloproteinases (Miyazaki et al, 1990) were underexpressed in MDR1 transfected clones. Cell adhesion is not only relevant for its direct association with drug resistance (Desoize et al, 1998) but also for its involvement in apoptosis (Damiano et al, 1999) and tumor suppression. In addition, the effect of cadherins on tissue morphogenesis suggests that the altered expression of adhesion-related genes could also be related to the biology and differential selection (Scotlandi et al, 1999) of cell clones.

Inhibition of apoptosis could be associated with an altered pro-apoptotic drug efflux by Pgp (Johnstone et al, 1999). MDR1 has also been associated directly with signaling pathways of apoptosis (Damiano et al, 1999; Johnstone et al, 1999). Data from our study reveals underexpression of signaling apoptotic targets such as I-TRAF, a tumor necrosis factor cascade effector (Kim and Gupta, 2000), and advillin, a gene recently related to programmed cell death (Marks et al, 1998). Accordingly, the percentage of apoptotic cells in U-2 OS was shown to be close to 0 , and that number did not significantly differ from the transfected clones (Scotlandi et al, 1999). Given that alterations in oncogene expression might affect cell-death pathways (Sinha et al, 1995), the possibility that differentially expressed oncogenes in our series, such as rasrelated proteins, might contribute to this inhibition of apoptosis should also be considered.

Staufen was underexpressed as a result of MDR1 transfection. This RNA binding protein is associated with both the essential RNA component and the catalytic subunit of human telomerase. Staufen has recently been proposed to play a role in telomerase activity, by affecting processing, assembly, or localization (Le et al, 2000). Because both telomere length and telomerase activity have been implicated in cellular senescence and cancer, this finding provides new insight into MDR phenomena, the clinical implications of which remain to be elucidated.

Expression changes of tumor-suppression related genes in MDR1 transfected cells included overexpression of the connector enhancer suppressor of ras (Therrien et al, 1998) and KISS-1, a metastasis suppressor gene (Lee et al, 1996). KISS-1 appears to be an interesting target because it regulates downstream events of cell-matrix adhesion involving cytoskeletal reorganization; it has also been reported overexpressed in nonmetastatic melanoma and breast models (Lee et al, 1996; Lee and Welch, 1997). Interest- 
ingly, WDNM-1, another metastasis-related gene, was also found to be underexpressed in our study (Lee et al, 1997).

The association of MDR1 with immune response targets such as cytokine oncostatin-M (Lu et al, 1993) and the recently described leukocyte complex receptor LENG2 (Wende et al, 2000) had not been reported to date. The underexpression of these genes might reflect a protective mechanism of the cancer cells against the host immune response. Taken together, the above-mentioned expression changes involving cellular trafficking, adhesion, apoptosis, senescence, tumor suppression, and immune system receptors suggest that MDR1 expression might be providing alternative cellular defense mechanisms leading to immortalization in resistant cancer cells.

The less resistant clone U-2/MDR117.1 was found to overexpress genes whose alterations have already been shown to be involved in MDR such as glutathione S transferase (Ueda et al, 1987), proteasome enzymes (Adams et al, 2000), and more recently, the kinesin heavy chain (Gudkov et al, 1994). The association of MDR1 with the tumor-progression-related tyrosine kinase receptor ligand ephrin (Tang et al, 1999) had not been reported to date. The higher MDR1 expression levels of U-2/DOX035 were associated with higher expression of adhesion-related genes, tumor progression related genes such as KISS-1, and immune-response receptors. None of these genes have previously been reported in osteosarcoma. Because these differences might account for differential degrees of resistance (Scotlandi et al, 1999), these genes represent potential targets for discriminating MDR patients more likely to progress. In osteosarcoma, MDR has been shown to be associated with a less aggressive phenotype, both in vitro and in a clinical setting (Chan et al, 1997; Scotlandi et al, 1999), and Pgp was postulated to have a causeeffect relationship with the reduced tumorigenic and metastatic potential of MDR cells (Biedler and Spengler, 1994; Scotlandi et al, 1999). This hypothesis could be supported by the fact that Pgp is highly expressed in certain mature, differentiated cells, and the tumors expressing Pgp might be the more differentiated lesions in their schemes of tumor progression (Biedler and Spengler, 1994; Gottesman and Pastan, 1993; Scotlandi et al, 1999; Ueda et al, 1987).

Gene-expression changes reported here are consistent with previous observations and provide confidence in our data. MDR1 transfection affected the three-dimensional cellular skeletal networks: the extracellular matrix (adhesion molecules), the cytoskeleton (actin), and the nuclear matrix (histone). This correlated well with the suggested involvement of the organization of actin filaments associated with cellular differentiation in Pgp function in certain MDR osteosarcoma cells (Takeshita et al, 1998). Alteration of genes related to alternative glucose metabolism such as phosphofructokinase or the UDP-glucose receptor may be related to the decreased dependance of the cancer cell on oxygen to generate energy (Schwartz and Beitner, 2000). Expression changes of genes coding for intracellular $\mathrm{pH}$ regulators support altered partitioning MDR models (Roepe, 2000) and the association of intracellular $\mathrm{H}+$ concentrations with apoptosis (Voehringer et al, 2000). Expression of HIV-related genes support the involvement of Pgp in HIV infection (Lee et al, 2000). Finally, RNA polymerase, the ubiquitin-proteasome system, and TRAF-I expression levels are consistent with a recent microarray study evaluating the effects of doxorubicin treatment (Kudoh et al, 2000). However, it is not clear whether these genes are directly related to MDR1 or secondarily to any of the genes directly expressed by MDR1.

The manipulation of cells by NEO and selection with the antibiotic neomycin appears to provoke expression changes within the cells. Expression changes observed between $U-2 / N E O 8$ versus $U-2$ OS cells can be related to the known RNA-binding capacity of the aminoglycoside neomycin. Neomycin can induce misreading of the genetic code, inhibiting or potentiating catalytic RNAs, and interfering with cellular proliferation and programmed cell death (Schroeder et al, 2000). Nevertheless, several reasons support considering U-2/NEO8 cells as a control in our experimental design. First, U-2/NEO8 cells displayed an MDRnegative phenotype (Scotlandi et al, 1999). Second, an optimal hybridization was confirmed throughout the dynamic range in all microarrays and Figure 4 displays the experiment comparing NEO transfected clones versus the parental cells (Callow et al, 2000). In addition, by comparing NEO and MDR1 transfected cells, we found that most of the commonly expressed genes may be accounted for by the RNA-binding ability of neomycin and clone differentiation rather than by the MDR phenotype itself. Finally, hierarchical clustering analysis showed that cells transfected with MDR1 displayed more related expression profiles than NEO transfected clones.

This study provides a more comprehensive view of the global effects of MDR1 expression by identifying genes involved in MDR related to multiple processes. It also provides a framework for future approaches to characterization of the differentially expressed genes that might be involved in the clinical phenomenon of MDR. The understanding of these transcriptional changes may contribute to translational strategies in predicting resistance and to the development of new therapeutic interventions.

\section{Materials and Methods}

\section{Cell Culture and Transfection}

U-2 OS human osteosarcoma cells were maintained in Iscove modified Dulbecco medium supplemented with penicillin $(100 \mathrm{U} / \mathrm{ml})$, streptomycin $(100 \mu \mathrm{g} / \mathrm{ml})$, and $10 \%$ heat inactivated $\mathrm{FCS}$ at $37^{\circ} \mathrm{C}$ in a humidified $5 \% \mathrm{CO}_{2}$ atmosphere. The transfection of $\mathrm{U}-2$ OS was performed 24 hours after seeding of $10^{5}$ cells in $100-\mathrm{mm}^{2}$ dishes using a standard calcium phosphate technique. U-2/DOXO35 and U-2/DOXO23 clones were obtained by transfection with $10 \mu \mathrm{g}$ of pFR$\mathrm{CMV}$, an expression vector containing a full-length 
MDR1 cDNA for 24 hours. After 2 days in their regular medium, cultures were exposed to selective medium containing $300 \mathrm{ng} / \mathrm{ml}$ of doxorubicin and refed every other day for 2 weeks to select resistant colonies. To evaluate the expression changes associated with MDR1 transfection independently of the selection agent used, U-2/MDR117.1 and U-2/MDR117.2 clones were generated by cotransfecting U-2 OS cells with $10 \mu \mathrm{g}$ of pFR-CMV and $1 \mu \mathrm{g}$ of pSV2neo and selected in medium containing $500 \mathrm{mg} / \mathrm{ml}$ of the neomycin analog G418. These pairs of MDR1 transfected and cotransfected subclones were selected based on their different but comparable MDR1 expression levels displayed (Scotlandi et al, 1999). The control clone U-2/NEO8 was obtained by transfection with calf thymus DNA and pSV2neo and selection with G418 (500 mg/ml). All cells were maintained for four to eight passages and harvested with trypsin- EDTA at 75 to $90 \%$ confluence.

\section{cDNA Clones and Construction of Microarrays}

Microarrays were constructed at the AECOM microarray facility (Cheung et al, 1999). The arrays contained a set of 8976 sequence-verified human GEMarray clones (Incyte/Genome Systems, Palo Alto, California) representing known genes and ESTs. Sequence verification is being performed on regular basis to validate the identity of the cDNA sequences on our arrays. We have observed only a $5 \%$ error rate out of 50 clones that have been directly sequence reverified.

\section{Hybridization Protocols}

Total RNA was extracted using the RNeasy protocol (Qiagen, Hilden, Germany). $10 \mu \mathrm{g}$ of total RNA were converted into fluorescent cDNA and hybridized according to the 3DNA Genisphere protocol (Montvale, New Jersey) (Stears et al, 2000). We have carried out one duplicate or one reverse-labeling experiment for validation of the differential expression profiles among different transfected cell clones. The average of these experiments has been used for the analysis of the results.

\section{Image Acquisition and Analysis}

Fluorescence intensities of the immobilized probes were determined from images generated either by a custom-built laser scanner (Cheung et al, 1999) or an Axon automated laser scanner (Axon Instruments, Foster City, California). Gridding of the arrays was performed using either the Scanalyze (Eisen et al, 1998), or the GenePix software (Axon Instruments).

Before any analysis, plots of the fold change versus the average intensity were examined to look for abnormalities in single-array data. It is common to plot a red versus green channel scatter plot to examine distribution of intensities; however, we found that transforming to fold change versus average intensity displayed the data in a more easily viewed form. If Ired is the background-subtracted red-channel intensity, and Igreen is the background-subtracted green inten- sity, then the following variables were created: $R=$ Ired/Igreen and $A=V$ (Ired $\times$ Igreen), where $R$ is simply the fold-change ratio, and $A$ is the average intensity (the geometric mean which is equivalent to averaging the log intensity). The curvature in the scatter plot indicated a dependence of the ratio $\mathrm{R}$ on the overall intensity. This curve is then used to normalize the data: loglred/Igreen -> log (Ired/Igreen) $c(A)$ where $c(A)$ is the fit. This is equivalent to multiplying the green-channel intensity (or dividing the red) by an intensity-dependent normalization constant $k(A)$ where $\log [(k(A)]=c(A)$. Optimal normalized data should be horizontal and centered at 0 (Callow et al, 2000). Samples were normalized using this intensitydependent normalization, which is more accurate than normalization with a fixed constant. Normalized fold changes in gene expression were then used to further analyze and cluster the various cell lines.

\section{Statistical Analysis}

A critical issue in microarray data analysis is to define an optimal cutoff level to consider a differential expression as discriminative. Given the fact that the derived clones were obtained from transfection-cotransfection of the same parental cell line, we selected a stringent cutoff of a 2-fold fluorescent ratio, and only genes showing intensities higher than 500 arbitrary fluorescence units in at least one of the channels were considered for further analysis. The entire data set of genes showing red $(R)$ to green $(G)$ and $G / R$ ratios higher than 2.0 in each experiment has been tabulated will be available at the web site created for this manuscript at www.mskcc.org/GCL/ SarcomaGenomics.

Hierarchical clustering was performed to explore the relationships among the cell lines and represent them in the resulting dendrogram. Average linkage clustering using the Spearman coefficient test was applied using only those genes that provided data in all cell lines. Only the 226 genes showing R/G ratios of 2-fold or higher in at least one experiment were considered for clustering analysis (Eisen et al, 1998). Only sequence assigned ESTs and genes with known function at the time of blasting are commented upon in this manuscript.

Validation of the Results: Northern Blotting, Immunohistochemistry, and Semiquantitative PCR. Northern hybridization of certain interesting differentially expressed genes was performed by standard methods (these will be available in our web site). Total RNA, $10 \mu \mathrm{g}$ per lane, was fractionated in $1.2 \%$ agaroseformaldehyde gels by electrophoresis. RNA was transferred onto nylon membranes by capillary blotting overnight. The clones were grown, and cDNA fragments were isolated using Qiagen columns and restriction enzymes (Valencia, California). The probes were labeled with $\left[{ }^{32}-\mathrm{P}\right]$ deoxycytidine triphosphate using random priming. Northern hybridization was performed at $42^{\circ} \mathrm{C}$ overnight in $10 \mathrm{~mL}$ hybridization buffer including $25 \mathrm{ng}$ of the labeled probe. The membranes were washed in $0.2 \times \mathrm{SSC}$ at $24^{\circ} \mathrm{C}$ for 20 minutes. Blots were exposed to Kodak X-Omat AR 
film (New Haven, Connecticut) at $-80^{\circ} \mathrm{C}$ for 2 hours to 4 days.

The differential protein expression of MDR1 was additionally confirmed by immunohistochemistry and performed on cytospins obtained from all cell clones using anti-Pgp mAb clone HYB241 (Cordon-Cardo et al, 1990). Ephrin B3 expression was confirmed by immunohistochemical analysis using a purified goat serum (AF395; R\&D Systems, Minneapolis, Minnesota). EPCAM expression was also confirmed by immunohistochemical analysis using a mouse mAb (B302 [323/A3]; R\&D Systems). Biotinylated horse antimouse and rabbit antigoat antibodies (1:500 dilution, incubation 30 minutes; Vector Laboratories, Burlingame, California) were used as secondary reagents. Immunoreactivities were visualized by avidin/biotin peroxidase conjugated complexes (1:25 dilution, incubation 30 minutes; Vector Laboratories) and diaminobenzidine tetrahydrochloride as the final chromogen. Counterstaining was performed with hematoxylin.

Semiquantitative PCR was performed for the KISS-1 gene using RNA extracted with TRIzol (Invitrogen, Carlsbad, California) from the U-2 OS parental cell line and MDR transfectants. cDNA was obtained from $1 \mu \mathrm{g}$ of RNA by RT-PCR standard methods using Moloney murine leukemia virus reverse transcriptase (Invitrogen) in the presence of dNTPs and oligo-dT. Semiquantitative PCR was performed using $2 \mu \mathrm{g}$ of cDNA and the following amplimers: 5'-AGGACCTGGCTCTTCTCACCA-3' and 5'-AGCAGCTGGCTTCTTCTCG-3'. The reaction was carried out for $20,24,27$, and 30 cycles, with a $60^{\circ} \mathrm{C}$ annealing temperature. The PCR product is a fragment of 202 bp, separated by electrophoresis on a $2 \%$ agarose gel. The intensity of the amplified product in the different U-2 OS clones was determined by using an image analysis system (Quantity One system; BioRad, Hercules, California) and normalized to the relative value of the $\beta$-actin product (control gene).

\section{Acknowledgments}

The authors thank Maria Dudas, Elizabeth Charytonowicz, Thomas Harris, Nicholas D. Socci and Aldo Massimi for their technical support and Kathleen Scotto for critical review of the manuscript.

\section{References}

Adams J, Palombella VJ, and Elliott PJ (2000). Proteasome inhibition: A new strategy in cancer treatment. Invest New Drugs 18:109-121.

Biedler JL and Spengler BA (1994). Reverse transformation of multidrug-resistant cells. Cancer Metastasis Rev 13:191-207.

Callow MJ, Dudoit S, Gong EL, Speed TP, and Rubin EM (2000). Microarray expression profiling identifies genes with altered expression in HDL-deficient mice. Genome Res 10: 2022-2029.

Chambers JK, Macdonald LE, Sarau HM, Ames RS, Freeman K, Foley JJ, Zhu Y, McLaughlin MM, Murdock P, McMillan L, Trill J, Swift A, Aiyar N, Taylor P, Vawter L, Naheed S, Szekeres P, Hervieu G, Scott C, Watson JM, Murphy AJ, Duzic E, Klein C,
Bergsma DJ, Wilson S, and Livi GP (2000). A G protein-coupled receptor for UDP-glucose. J Biol Chem 275:10767-10771.

Chan HS, Grogan TM, Haddad G, DeBoer G, and Ling V (1997). P-glycoprotein expression: Critical determinant in the response to osteosarcoma chemotherapy. J Natl Cancer Inst 89:1706-1715.

Cheung VG, Morley M, Aguilar F, Massimi A, Kucherlapati R, and Childs $G$ (1999). Making and reading microarrays. Nat Genet 21:15-19.

Cordon-Cardo C, O'Brien JP, Boccia J, Casals D, Bertino JR, and Melamed MR (1990). Expression of the multidrug resistance gene product (P-glycoprotein) in human normal and tumor tissues. J Histochem Cytochem 38:1277-1287.

Damiano JS, Cress AE, Hazlehurst LA, Shtil AA, and Dalton WS (1999). Cell adhesion mediated drug resistance (CAM$\mathrm{DR})$ : Role of integrins and resistance to apoptosis in human myeloma cell lines. Blood 93: 1658-1667.

Dano K (1973). Active outward transport of daunomycin in resistant Ehrlich ascites tumor cells. Biochim Biophys Acta 323:466-483.

Desoize B, Gimonet D, and Jardillier JC (1998). Multicellular resistance: Another mechanism for multidrug resistance? Bull Cancer 85:785.

Eisen MB, Spellman PT, Brown PO, and Botstein D (1998). Cluster analysis and display of genome-wide expression patterns. Proc Natl Acad Sci USA 95:14863-14868.

Gottesman MM and Pastan I (1993). Biochemistry of multidrug resistance mediated by the multidrug transporter. Annu Rev Biochem 62:385-427.

Gudkov AV, Kazarov AR, Thimmapaya R, Axenovich SA, Mazo IA, and Roninson IB (1994). Cloning mammalian genes by expression selection of genetic suppressor elements: Association of kinesin with drug resistance and cell immortalization. Proc Natl Acad Sci USA 91:3744-3748.

Hirayama S, Bujo H, Yamazaki H, Kanaki T, Takahashi K, Kobayashi J, Schneider WJ, and Saito Y (2000). Differential expression of LR11 during proliferation and differentiation of cultured neuroblastoma cells. Biochem Biophys Res Commun 275:365-373.

Hirota T, Morisaki T, Nishiyama Y, Marumoto T, Tada K, Hara T, Masuko N, Inagaki M, Hatakeyama K, and Saya H (2000). Zyxin, a regulator of actin filament assembly, targets the mitotic apparatus by interacting with h-warts/LATS1 tumor suppressor. J Cell Biol 149:1073-1086.

Johnstone RW, Cretney E, and Smyth MJ (1999). P-glycoprotein protects leukemia cells against caspasedependent, but not caspase-independent, cell death. Blood 93:1075-1085.

Kim CH and Gupta S (2000). Expression of TRAIL (Apo2L), DR4 (TRAIL receptor 1), DR5 (TRAIL receptor 2) and TRID (TRAIL receptor 3 ) genes in multidrug resistant human acute myeloid leukemia cell lines that overexpress MDR 1 (HL60/ Tax) or MRP (HL60/AR). Int J Oncol 16:1137-1139.

Kudoh K. Ramanna M, Ravatn R, Elkahloun AG, Bittner ML, Meltzer PS, Trent JM, Dalton WS, and Chin KV (2000). Monitoring the expression profiles of doxorubicin-induced and doxorubicin-resistant cancer cells by cDNA microarray. Cancer Res 60:4161-4166.

Le S, Sternglanz R, and Greider CW (2000). Identification of two RNA-binding proteins associated with human telomerase RNA. Mol Biol Cell 11:999-1010. 
Lee CG, Ramachandra M, Jeang KT, Martin MA, Pastan I, and Gottesman MM (2000). Effect of ABC transporters on HIV-1 infection: Inhibition of virus production by the MDR1 transporter. FASEB J 14:516-522.

Lee JH, Miele ME, Hicks DJ, Phillips KK, Trent JM, Weissman BE, and Welch DR (1996). KiSS-1, a novel human malignant melanoma metastasis-suppressor gene. J Natl Cancer Inst 88:1731-1737.

Lee JH and Welch DR (1997). Suppression of metastasis in human breast carcinoma MDA-MB-435 cells after transfection with the metastasis suppressor gene, KiSS-1. Cancer Res 57:2384-2387.

Lee M, Hwang I, Choi Y, Paik S, Lee HB, and Baik M (1997). EGF inhibits expression of WDNM1 and sulfated glycoprotein-2 genes in mammary epithelial cells. Biochem Biophys Res Commun 240:104-107.

Lu C, Rak JW, Kobayashi H, and Kerbel RS (1993). Increased resistance to oncostatin $\mathrm{M}$-induced growth inhibition of human melanoma cell lines derived from advanced-stage lesions. Cancer Res 53:2708-2711.

Marks PW, Arai M, Bandura JL, and Kwiatkowski DJ (1998). Advillin (p92): A new member of the gelsolin/villin family of actin regulatory proteins. J Cell Sci 111:2129-2136.

Miyazaki K, Hattori Y, Umenishi F, Yasumitsu H, and Umeda $M$ (1990). Purification and characterization of extracellular matrix-degrading metalloproteinase, matrin (pump-1), secreted from human rectal carcinoma cell line. Cancer Res 50:7758-7764.

Modregger J, Ritter B, Witter B, Paulsson M, and Plomann M (2000). All three PACSIN isoforms bind to endocytic proteins and inhibit endocytosis. J Cell Sci 113:4511-4521.

Okazaki M, Takeshita S, Kawai S, Kikuno R, Tsujimura A, Kudo A, and Amann E (1994). Molecular cloning and characterization of OB-cadherin, a new member of cadherin family expressed in osteoblasts. J Biol Chem 269:12092-12098.

Roepe PD (2000). What is the precise role of human MDR 1 protein in chemotherapeutic drug resistance? Curr Pharm Des 6:241-260.

Schinkel AH, Mayer U, Wagenaar E, Mol CA, van Deemter L, Smit JJ, van der Valk MA, Voordouw AC, Spits $H$, van Tellingen O, Zijlmans JM, Fibbe WE, and Borst P (1997). Normal viability and altered pharmacokinetics in mice lacking mdr1-type (drug-transporting) P-glycoproteins. Proc Natl Acad Sci USA 94:4028-4033.

Schroeder R, Waldsich C, and Wank H (2000). Modulation of RNA function by aminoglycoside antibiotics. EMBO J 19:1-9.

Schwartz D and Beitner R (2000). Detachment of the glycolytic enzymes, phosphofructokinase and aldolase, from cytoskeleton of melanoma cells, induced by local anesthetics. Mol Genet Metab 69:159-164.

Scotlandi K, Manara MC, Serra M, Benini S, Maurici D, Caputo A, De Giovanni C, Lollini PL, Nanni P, Picci P, Campanacci M, and Baldini N (1999). The expression of P-glycoprotein is causally related to a less aggressive phenotype in human osteosarcoma cells. Oncogene 18:739-746.

Scotlandi K, Serra M, Nicoletti G, Vaccari M, Manara MC, Nini G, Landuzzi L, Colacci A, Bacci G, Bertoni F, Picci P, Campanacci M, and Baldini N (1996). Multidrug resistance and malignancy in human osteosarcoma. Cancer Res 56: 2434-2439.
Sinha BK, Yamazaki H, Eliot HM, Schneider E, Borner MM, and O'Connor PM (1995). Relationships between proto-oncogene expression and apoptosis induced by anticancer drugs in human prostate tumor cells. Biochim Biophys Acta 1270:12-18.

St. Croix B and Kerbel RS (1997). Cell adhesion and drug resistance in cancer. Curr Opin Oncol 9:549-556.

Stears RL, Getts RC, and Gullans SR (2000). A novel, sensitive detection system for high-density microarrays using dendrimer technology. Physiol Genomics 3:93-99.

Takeshita H, Kusuzaki K, Ashihara T, Gebhardt MC, Mankin $\mathrm{HJ}$, and Hirasawa $Y$ (1998). Actin organization associated with the expression of multidrug resistant phenotype in osteosarcoma cells and the effect of actin depolymerization on drug resistance. Cancer Lett 126:75-81.

Tang XX, Evans AE, Zhao H, Cnaan A, London W, Cohn SL, Brodeur GM, and Ikegaki N (1999). High-level expression of EPHB6, EFNB2, and EFNB3 is associated with low tumor stage and high TrkA expression in human neuroblastomas. Clin Cancer Res 5:1491-1496.

Taniguchi M, Miura K, Iwao H, and Yamanaka S (2001). Quantitative assessment of DNA microarrays: Comparison with Northern blot analyses. Genomics 71:34-39.

Tenenhouse HS (1999). Recent advances in epithelial sodium-coupled phosphate transport. Curr Opin Nephrol Hypertens 8:407-414.

Therrien M, Wong AM, and Rubin GM (1998). CNK, a RAF-binding multidomain protein required for RAS signaling. Cell 95:343-353.

Trebak M, Begg GE, Chong JM, Kanazireva EV, Herlyn D, and Speicher DW (2001). Oligomeric state of the colon carcinoma-associated glycoprotein GA733-2 (Ep-CAM/ EGP40) and its role in GA733-mediated homotypic cell-cell adhesion. J Biol Chem 276:2299-2309.

Ueda K, Cardarelli C, Gottesman MM, and Pastan I (1987). Expression of a full-length cDNA for the human "MDR1" gene confers resistance to colchicine, doxorubicin, and vinblastine. Proc Natl Acad Sci USA 84:3004-3008.

Voehringer DW, Hirschberg DL, Xiao J, Lu Q, Roederer M, Lock CB, Herzenberg LA, Steinman L, and Herzenberg LA (2000). Gene microarray identification of redox and mitochondrial elements that control resistance or sensitivity to apoptosis. Proc Natl Acad Sci USA 97:2680-2685.

Wende H, Volz A, and Ziegler A (2000). Extensive gene duplications and a large inversion characterize the human leukocyte receptor cluster. Immunogenetics 51:703-713.

Wunder JS, Bull SB, Aneliunas V, Lee PD, Davis AM, Beauchamp CP, Conrad EU, Grimer RJ, Healey JH, Rock MJ, Bell RS, and Andrulis IL (2000). MDR1 gene expression and outcome in osteosarcoma: A prospective, multicenter study. J Clin Oncol 18:2685-2694.

Yue $\mathrm{H}$, Eastman PS, Wang BB, Minor J, Doctolero $\mathrm{MH}$, Nuttall RL, Stack R, Becker JW, Montgomery JR, Vainer M, and Johnston $R$ (2001). An evaluation of the performance of cDNA microarrays for detecting changes in global mRNA expression. Nucleic Acids Res 29:E41-1. 